Chem. Pharm. Bull.

29(2) 519-531 (1981)]

\title{
Studies on the Absorption of practically Water-insoluble Drugs following Injection. I. Intramuscular Absorption from Water- immiscible Oil Solutions in Rats
}

\author{
Koichiro Hirano,* Teruhisa Ichihashi, and Hideo Yamada \\ Shionogi Research Laboratories, Shionogi and Co., Ltd., Sagisu 5-12-4, \\ Fukushima-ku, Osaka, 533, Japan
}

(Received July 30, 1980)

\begin{abstract}
The absorption behavior following intramuscular injection of practically waterinsoluble drugs in water-immiscible oil solution was investigated with several azo dyes and two steroids as model drugs by the local clearance method in the $m$. gastrocnemius of the rat. The absorption of the drug component obeyed approximately 1st order kinetics, while the absorption of the oily solvent was very slow. The injection volume $\left(V_{0}\right)$ influenced the absorption rate constant $(k)$ and the correlation $k \propto V_{0}{ }^{m}$ was experimentally observed (for intact rats, $m=-0.14$; for anesthetized rats, $m=-0.32$ ). This was also the case at another injection site, the $m$. rectus femoris in rats. Comparison of the absorption rate of a drug in various oil vehicles showed that $k$ was controlled predominantly by the oil/water distribution coefficient $(K)$ and depended little on the viscosity of the vehicle. These results suggested that the release process of the drug component from the oily depot to the aqueous phase around it was the main route for absorption, and that the subsequent transport process in the aqueous phase might be rate-limiting. A plot of $\log k$ versus $\log K$ gave a straight line with a slope close to -1 , but of a slightly smaller absolute value. This plot could be applied satisfactorily for estimating the absorption rate of other drug-oil systems. A guideline for predicting the absorption rate of a drug in oily suspension from the $k$ value for its solution is presented.
\end{abstract}

Keywords_-drug absorption kinetics; intramuscular injection; local clearance method; practically water-insoluble drugs; water-immiscible oil vehicles; injection volume; distribution coefficient; intact and anesthetized rats

Parenteral routes of drug administration are as useful and important as oral ones for early screening and preclinical testing of drugs in animals. There are many kinds of injections, including intramuscular, subcutaneous, intravenous, intradermal, hypodermal, intraarterial, intrapleural, intraperitoneal, intraarticular, intracardial, intraspinal and intracerebral. $\mathrm{Ab}-$ sorption is not involved when a drug is administered parenterally by an intravasular route. However, when the drug is administered by any extravascular route, a depot of some type is formed and the drug must leave the depot and reach the blood or lymph systems by some process (es).

Since the publication of several reviews ${ }^{1)}$ there has been increasing interest in the absorption behavior of drugs in aqueous solution from intramuscular ${ }^{2}$ and subcutaneous sites. ${ }^{3)}$ However, compared to water-soluble drugs, little work has been done on practically waterinsoluble drugs. These drugs are commonly administered to laboratory animals as parenteral preparations in the form of oily solutions (oily suspensions), aqueous suspensions, aqueous solutions solubilized with nonionic surfactants, or emulsions. The absorption of such drugs, which depends on the dosage form or formulation, is in most cases slow enough to be ratedeterminant in their disposition in the body. This means that even pharmacological responses are sometimes governed primarily by the formulation itself. Therefore, basic investigation on drug absorption from such dosage forms by each parenteral route is required in order to select the optimal preparation and parenteral route for more complete screening tests in animals.

The present study was undertaken to elucidate the absorption behavior of practically water-insoluble drugs from water-immiscible oil solutions injected intramuscularly. Early 
investigators ${ }^{4}$ noted mainly the vehicle effect on the pharmacologic responses or blood levels of drugs but did not refer to the mechanism or kinetics of drug absorption from such preparations. Recently, Tanaka et al. ${ }^{5}$ ) studied these problems using slightly water-soluble drugs in anesthetized and operated rats.

For our study, several azo dyes and some steroids which are unionized and practically water-insoluble under physiological conditions were used as model drugs, and their intramuscular absorption properties were examined by the local clearance method in the $m$. gastrocnemius of the intact rat. Under various experimental systems and conditions, the intramuscular absorption rates of these model drugs were compared to evaluate the contribution of physicochemical factors and to clarify the kinetic process. On the basis of the results, predictions of the absorption rates of other drugs in oily solutions were attempted. The relationship between absorption rates in oily solution and oily suspension was also investigated.

\section{Experimental}

Materials-Azo dyes such as p-aminoazobenzene (PAAB), p-hydroxyazobenzene (PHAB), o-aminoazotoluene (OAAT), 1-phenylazo-2-naphthylamine (PANA) and tetrazobenzene- $\beta$-naphthol (Sudan III), and steroids such as testosterone (TS) and $2 \alpha, 3 \alpha$-epithio- $5 \alpha$-androstan- $17 \beta$-ol (epitiostanol) were selected as model compounds for practically water-insoluble drugs. These compounds (other than epitiostanol) were obtained commercially: PAAB, PANA and TS, Tokyo Kasei Kogyo Co., Ltd. (Tokyo); PHAB, Eastman Kodak Co. (N.Y.); OAAT, Ishizu Pharmaceutical Co., Ltd. (Osaka); Sudan III, E. Merck AG (Darmstadt). These compounds were of reagent grade and were used without further purification, except for PHAB, which was purified by being dissolved in $\mathrm{MeOH}$ then recrystallized with water. Epitiostanol was synthesized in our laboratory and was of medicinal grade. As water-immiscible oil solvents for the injection preparations, sesame oil (SO), medium chain $\left(\mathrm{C}_{8}-\mathrm{C}_{12}\right)$ triglyceride commercially named Miglyol 812 (Mig), isopropyl myristate (IPM), diethyl sebacate (DES) and castor oil (CO) were selected in view of relatively wide variety in viscosity and dissolving powder. These compounds were also obtained from commercial sources: SO, Maruishi Pharmaceutical Co., Ltd. (Osaka); Mig, Chemische Werke Witten (Germany); IPM and DES, Nikko Chemicals Co., Ltd. (Tokyo); CO, Kenei Pharmaceutical Co. (Japan). SO and CO were of JP grade and the others were of reagent grade. All were used without further purification. All other chemicals used in this investigation were of analytical or reagent grade.

Test Injection Preparations--An oily solution was prepared by dissolving the desired amount of a test compound in an oily solvent and filtering it through an SM 116 membrane filter (Sartorius-Membranfilter $\mathrm{GmbH}$, Göttingen). All test solutions used here were ascertained to be chemically and physically stable for at least the experimental period. An oily suspension of PAAB or TS was formulated in a room held at $37^{\circ}$ by dispersing the sieved powder (for PAAB, 74-149 $\mu \mathrm{m}$ in diameter; for TS, 37-74 and $74-149 \mu \mathrm{m}$ ) in the oil vehicle presaturated with each drug using a mixer (Micro Thermo Mixer Model TM-101, Thermonics Co., Ltd., Tokyo), and the product was stored at $37^{\circ}$ until use. All suspensions were used about $20 \mathrm{hr}$ after preparation.

Animal Experiments-_-Male Wistar albino rats weighing $250 \pm 30$ g were used in all animal experiments.

(i) Absorption Experiment Procedure: The injection site for the present study, unless otherwise mentioned, was the medial head of the $m$. gastrocnemius in the $m$. triceps surae of the left hind leg in the rat; this site was selected because the location of the needle tip could be easily controlled. The absorption time course was followed by the local clearance method. The rat was given light anesthesia with ether, then a thin Terumo needle $\left(27 \mathrm{G} \times 3 / 4^{\prime \prime}\right.$ for oily solutions and $25 \mathrm{G} \times 1^{\prime \prime}$ for oily suspensions; Terumo Co., Ltd., Tokyo) connected to a volume scale-corrected syringe or Terumo micrometer syringe (MS 10, 50 and 100) was inserted a few millimeters above the ankle and the tip of the needle was led to the center of the m. gastrocnemius, medial head. Next, the test solution (50 $\mu \mathrm{l}$, unless otherwise stated) was injected at moderate speed. Immediately after withdrawal of the needle, the quick adhesive Aron Alpha (Toa Gousei Kagaku Kogyo Co., Ltd., Tokyo) was applied to the insertion site in order to prevent leakage of the oily solution injected. During the absorption experiment, the rat was housed in a cage which allowed free movement and easy access to water and food. At various intervals after the injection, rats were decapitated and bled, and the muscle tissues around the injection site, including the oily depot, were excised as completely as possible. The removed muscles were mixed with $5 \mathrm{ml}$ of water and homogenized with a high speed blender (Ultra-Turrax, Janke and Kunkel K.G., Ger.) under cooling in an ice-water bath. Another $5 \mathrm{ml}$ of water was used to wash the blender of the homogenizer and this was added to the above homogenate. After extraction from the homogenate with $8 \mathrm{ml}$ of ethyl acetate, the residual amount of the drug in the removed muscles was analyzed. For absorption studies with sesame oil (SO), the following indirect method was adopted. Fifty microliters of SO solution containing Sudan III $(5 \mathrm{mg} / \mathrm{ml})$ was administered to the same site in the manner described above in two groups of rats. : At a set time interval, both groups were sacrificed. 
From one group, the residual amount $(W)$ of Sudan III was determined, and from the other, its concentration $(C)$ in SO solution remaining in the muscle was determined. Since Sudan III was pooled and kept homogeneously dissolved in the SO depot for at least $20 \mathrm{hr}$ after injection, the volume $(V)$ of SO remaining in the muscle could be approximately calculated as $V=W / C$.

(ii) In Vitro Incubation Experiment: In order to ascertain whether the clearance of a given drug from the injection site could be attributed to its transport into the vascular systems or not, an in vitvo incubation experiment was undertaken along with the absorption experiment, and the metabolic change at the injection site was checked. Fifty microliters of SO solution containing $0.5 \mathrm{mg} / \mathrm{ml}$ of an azo compound was injected into the same site in the manner described above in two groups of rats. For one group, the muscles, including the injected solution, were removed immediately after administration, then incubated in $5 \mathrm{ml}$ of Ringer-Locke solution under bubbling with air and mild shaking at $37^{\circ}$ for 2 or $6 \mathrm{hr}$ (Yamato incubator, model BT-41; Yamato Scientific Co., Ltd., Tokyo). After incubation, the residual amount of the test compound was analyzed and compared with that obtained from the absorption experiment in another group of rats. Visible absorption spectra were also measured before and after the above experiments with a $\mathrm{Hitachi}$ EPS-3T recording spectrophotometer (Hitachi Co., Ltd., Tokyo) to check for metabolic change of the test compounds.

Measurement of Absolute Viscosity_-Kinematic viscosities of oily solvents were determined with an Ubbelohde viscometer (Kaburagi Kagaku Kikai Kogyo Co., Ltd., Japan) and their densities were measured by using a volume scale-corrected Cassia flask. These determinations were performed at $37^{\circ}$, and the absolute viscosity was calculated from the kinematic viscosity and density.

Determination of the Apparent Distribution Coefficient-Ten milliliters of $0.9 \%(\mathrm{w} / \mathrm{v}) \mathrm{NaCl}$ aqueous solution (saline) was added to $4 \mathrm{ml}$ of an oily solution containing the model compound (concentration: for PAAB, PHAB and OAAT, 5-40 mg/ml; for TS, $2.33-4.65 \mathrm{mg} / \mathrm{ml}$; for epitiostanol, $2-5 \mathrm{mg} / \mathrm{ml}$ ) and shaken well at $37^{\circ}$ with a mechanical shaker until distribution equilibrium was reached. Our preliminary experiment had demonstrated that $2 \mathrm{hr}$ was needed for this equilibrium, and that the volume change of each phase after equilibrium was small enough to neglect. After equilibrium had been reached, the unstable emulsion was centrifuged at $4000 \mathrm{rpm}$ for $10 \mathrm{~min}$ to separate the transparent aqueous phase, part of which was withdrawn for analysis. The concentration of the model drug in the oily phase was calculated from its concentration determined for the aqueous phase and its initial concentration in the oily phase. The apparent distribution coefficient $(K)$ was represented as the concentration ratio between the two phases. The value of $K$ as determined above depended little on the concentration and was similar to the solubility ratio at $37^{\circ}$ between the oil and saline solvents. For PANA, whose concentration in saline was too low to be determined accurately, the solubility ratio between the two solvents was used as the apparent distribution coefficient.

Analytical Method__-(i) Samples from Animal Experiments: Sample ethyl acetate solutions containing azo compounds were adequately diluted with ethyl acetate and their optical densities were measured with a Hitachi UV-VIS spectrophotometer at $384,348,386,450$ and $510 \mathrm{~nm}$ for PAAB, PHAB, OAAT, PANA and Sudan III, respectively. Epitiostanol was determined by GLC after being transformed to olefin (5 $\alpha$ androst-en-17 $\beta$-ol) in the following manner. ${ }^{6}$ ) Five milliliters of sample solution (epitiostanol content, $0-160 \mu \mathrm{g}$ ) was shaken with $1 \mathrm{~g}$ of $\mathrm{Al}_{2} \mathrm{O}_{3}$ (active, neutral, I, E. Merck, Darmstadt) for 10 min and centrifuged at $3000 \mathrm{rpm}$ for $5 \mathrm{~min}$ (this procedure was done to minimize interfering materials in the following GLC assay). To $1.5 \mathrm{ml}$ of the supernatant was added $0.5 \mathrm{ml}$ of internal standard solution $(3 \beta$-acetoxy- $5 \alpha$-androstan in ethyl acetate, $0.25 \mathrm{mg} / \mathrm{ml}$ ), then the mixture was evaporated to dryness in a Vapour Mix instrument (Tokyo Rikakikai Co., Ltd., Tokyo). The residue was dissolved in $5 \mathrm{ml}$ of benzene and refluxed with about $30 \mathrm{mg}$ of spongy active copper $(\mathrm{Cu}-\mathrm{Zn})$ for $40 \mathrm{~min}$. The reaction mixture was filtered through Toyo filter paper No. 131 , and $2 \mathrm{ml}$ of the filtrate was evaporated to dryness. The residue was redissolved in about $200 \mu 1$ of ethyl acetate and $1-2 \mu \mathrm{l}$ of this was analyzed with a gas chromatograph, model GLC-4APTF, equipped with a flame ionization detector (Shimadzu Seisakusho, Ltd., Kyoto) under the following conditions. The column was a $1.5 \mathrm{~m} \times 5 \mathrm{~mm}$ glass tube packed with $3 \%$ SE-30 on Gas Chrom Q (80-100 mesh); carrier gas, $99.999 \% \mathrm{~N}_{2}$ (flow rate, $60 \mathrm{ml} / \mathrm{min}$ ); column, injection port and detector temperatures, 250, 268 and 288', respectively. In the case of TS, $3 \mathrm{ml}$ of sample solution was shaken with $0.6 \mathrm{~g}$ of an $\mathrm{Al}_{2} \mathrm{O}_{3}$-silica gel ${ }^{7}$ ) mixture $(1: 1, \mathrm{w} / \mathrm{w})$ for $10 \mathrm{~min}$ then centrifuged at $3000 \mathrm{rpm}$ for $5 \mathrm{~min}$ (this procedure was performed for the same reason as in the case of epitiostanol). To $1 \mathrm{ml}$ of the supernatant was added $100 \mu 1$ of internal standard solution ( $3 \beta$-acetoxy- $5 \alpha$-androstan in ethyl acetate, $0.5 \mathrm{mg} / \mathrm{ml})$. Two microliters of this mixture was used for GLC analysis as described above (column, injection port and detector temperatures were modified to 260,280 and $293^{\circ}$, respectively).

(ii) Samples from Other Experiments: (1) Test Compounds in Oily Solutions: Sample Solutions for azo compounds were analyzed colorimetrically after dilution with chloroform (PAAB, $372 \mathrm{~nm} ; \mathrm{PHAB}, 345$ $\mathrm{nm}$; OAAT, $376 \mathrm{~nm}$; PANA, $436 \mathrm{~nm}$; Sudan III, $520 \mathrm{~nm}$ ). Epitiostanol and TS were assayed by GLC as described above after dilution with ethyl acetate. (2) Test Compounds in Saline: Sample solutions of azo compounds and TS were analyzed spectrophotometrically as follows: PAAB and PHAB at 376 and $348 \mathrm{~nm}$, respectively, after dilution with distilled water; OAAT and PANA at 379 and $470 \mathrm{~nm}$, respectively, after 10/9-fold dilution with EtOH; TS at $241 \mathrm{~nm}$ after 10-fold dilution with EtOH. Epitiostanol was assayed by the GLC method described above after extraction with ethyl acetate. 


\section{Results}

\section{Comparison of Clearance of Model Compounds in the Injection Site between in Vitro Incubation} and in Vivo Absorption Experiments

To date various experimental procedures have been proposed and used to evaluate the absorption rate of drugs parenterally administered: (1) observation of changes in the pharmacological effect with time; (2) following the blood level and/or urinary excretion, and pharmacokinetic analysis of the data; (3) following drug clearance at the injection site (local clearance method). We adopted the local clearance method since a sample oily solution injected intramuscularly remains localized, allowing almost complete recovery of the remaining drug. Apparent elimination of the drug observed by the local clearance method sometimes results not only from its transport to the blood or lymph system but also from its metabolic change at the injection site. Hence, in order to check the contribution of the latter factor in the case of the model compounds, an in vitro incubation experiment with removed muscle tissues was undertaken and the resulting data of \% recovery for a given period were compared with those from the in vivo absorption experiment. Table I gives this comparison for each compound in the SO solution. For all compounds, the recovery was near $100 \%$ for the in vitro incubation experiment but considerably lower for the in vivo absorption experiment. Further, no significant change of the visible absorption spectrum was observed before and after these two experiments. These results showed that the metabolic change of these model compounds in the muscle was almost negligible and their apparent clearances observed here resulted mainly from transport into vascular systems. These findings support the view that the model compounds and the absorption experiment procedure adopted here are appropriate for analyzing the true absorption phenomena of practically water-insoluble drugs injected intramuscularly.

TABLE I. Comparison of \% Recovery between in Vitro Incubation with Removed Muscle Tissues and in Vivo Absorption Experimenta)

\begin{tabular}{|c|c|c|c|}
\hline \multirow{2}{*}{ Compound } & \multirow{2}{*}{ Time (hr) } & \multicolumn{2}{|c|}{$\%$ recovery $^{b)}$} \\
\hline & & In vitro & In vivo \\
\hline PHAB & 2 & $97.0(2.9)$ & $38.1(3.7)$ \\
\hline $\mathrm{PAAB}$ & 2 & $98.9(5.8)$ & $52.9(5.2)$ \\
\hline OAAT & 6 & $99.6(4.3)$ & $65.0(8.5)$ \\
\hline PANA & 6 & $100.0(5.7)$ & $91.3(4.2)$ \\
\hline Sudan III & 6 & $100.5(0.9)$ & $98.8(0.8)$ \\
\hline
\end{tabular}

a) Vehicle, $\mathrm{SO}$; initial concentration $\left(C_{0}\right), 0.5 \mathrm{mg} / \mathrm{ml}$; injection volume $\left(V_{0}\right), 0.05 \mathrm{ml}$.

$b$ ) Each value represents the mean of 3 experiments with the standard deviation in parentheses.

\section{Clearance of Sesame Oil from the Injection Site}

Various vegetable oils such as sesame oil, olive oil, cotton seed oil, peanut oil and castor oil are usually used as oily solvents for preparations. Sesame oil (SO) is most commonly used in Japan. Since these oils consist not of a single component but of various triglycerides such as olein, linolein, stearin, palmitin and myristin, and other natural products, direct evaluation of their absorption characteristics from the injection site seems impossible. Recently, some reports have appeared on intramuscular absorption of mineral oil, ${ }^{81}{ }^{14} \mathrm{C}$-methyl oleate, ${ }^{5)}$ and ${ }^{14} \mathrm{C}$-tripalmitin mixed with sesame oil. ${ }^{9)}$ All these investigations indicated that these oils are absorbed very slowly.

An oil vehicle may be eliminated from the injection site mainly via one or both of two routes: (a) absorption after being dissolved in the body fluids or transformed into some hydrophilic compounds and (b) direct absorption after division into micro-droplets. Table I shows that Sudan III, which could scarcely be released from the SO vehicle into the aqueous phase, 
was insignificantly absorbed for at least $6 \mathrm{hr}$ after injection. Thus, with Sudan III as a tracing material, the absorption of SO was examined by an indirect method, as described in "Experimental." The results appear in Table II. The residual volume $(V)$ of SO and the residual amount $(W)$ of Sudan III $6 \mathrm{hr}$ after injection had little changed from their initial values, but subsequently decreased gradually. This observation showed that SO was scarcely absorbed during the first several hours after injection, but that after this it might be very slowly absorbed, presumably via both routes mentioned above.

TABLE II. Absorption of Sesame Oil Estimated by the Indirect Method

\begin{tabular}{cccc}
\hline \hline Time $(\mathrm{hr})$ & $W(\mu \mathrm{g})^{a)}$ & $C(\mu \mathrm{g} / \mu \mathrm{l})^{b)}$ & $V(\mu \mathrm{l})^{c)}$ \\
\hline 0 & $272.0(2.1)$ & $5.07(0.01)$ & $53.6(0.4)$ \\
6 & $268.7(1.0)$ & $5.03(0.02)$ & $53.5(0.2)$ \\
20 & $242.9(7.3)$ & $5.00(0.13)$ & $48.6(1.9)$ \\
41 & $239.4(24.3)$ & $5.26(0.28)$ & $45.5(5.2)$ \\
\hline
\end{tabular}

a) Residual amount of Sudan III.

b) Sudan III concentration in the oily depot phase.

c) Calculated residual volume of SO. $V=W / C$. Each result is given as the mean of at least 3 experiments followed by the standard deviation in parentheses.

\section{Time Course of Drug Absorption and Effect of Initial Drug Concentration}

Fig. 1 shows a time course of intramuscular absorption of PAAB after injection of its SO solution. The initial concentration $\left(C_{0}\right)$ and injection volume $\left(V_{0}\right)$ are given in the legend. The upper figure (A) shows the value of the \% remaining on a linear scale while the lower one (B) uses a logarithmic scale. From the linearity shown in Fig. 1B, this compound was expected to be absorbed mono-exponentially, that is, according to a 1st order rate process. In order to confirm this, the effect of the initial concentration $\left(C_{0}\right)$ of PAAB in the SO solution on absorption was examined. Fig. 2 shows a comparison of time courses of PAAB absorption on a semilogarithmic scale from three solutions of different $C_{0}$. All these absorption profiles gave

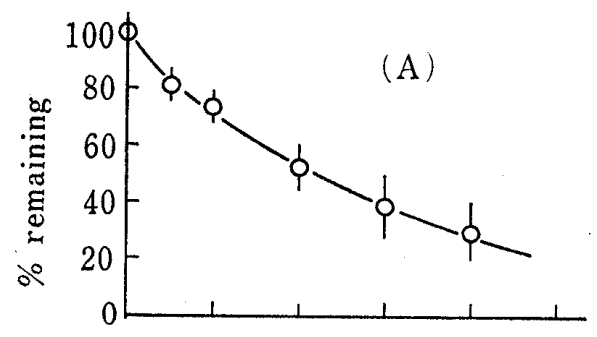

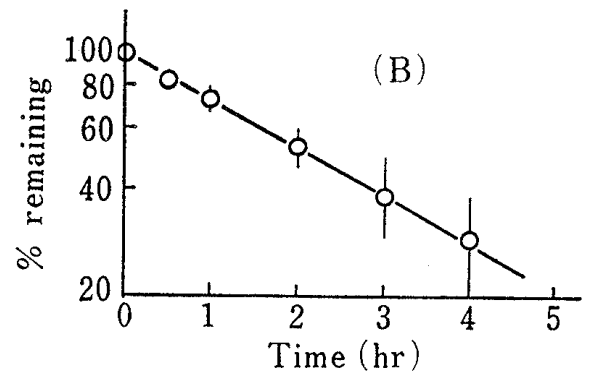

Fig. 1. Linear (A) and Semilogarithmic (B) Plots of \% Remaining PAAB at the Injection Site versus Time (SO)

Each point represents the mean of 4 experiments. The vertical bar about the mean shows the standard deviation. $C_{0}, 5 \mathrm{mg} / \mathrm{ml} ; V_{0}, 0.05 \mathrm{ml}$.

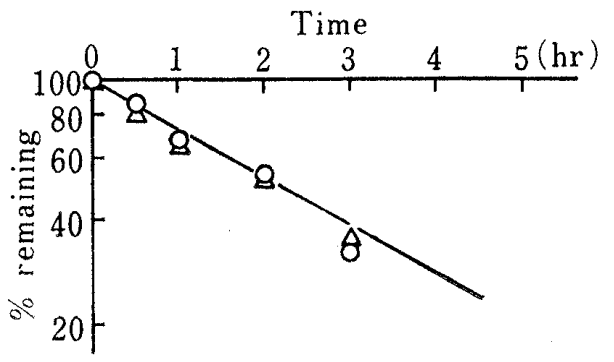

Fig. 2. Effect of Initial Concentration on PAAB Absorption following Intramuscular Injection (SO)

Key: $0,0.5 \mathrm{mg} / \mathrm{ml} ; \triangle 40 \mathrm{mg} / \mathrm{ml}$; solid line, $\tilde{5}$ $\mathrm{mg} / \mathrm{ml}$ (shown in Fig. 1B). Each point represents the mean of at least 3 experiments. $V_{0}: 0.05 \mathrm{ml}$. 
straight lines with the same slope, not depending on $C_{0}$. Other model compounds such as PHAB, OAAT and PANA in SO solution also showed similar behavior. These results were analogous to those obtained by Tanaka $e t$ al. ${ }^{5}$ ) who studied slightly water-soluble drugs and oil systems. Others have also reported that intramuscular absorption of neutral drugs in aqueous solution, different from that of acidic or basic drugs, depended little on their initial concentration. ${ }^{2 a, 10)}$ Therefore, it seems reasonable to conclude that intramuscular absorption of neutral and practically water-insoluble drugs in water-immiscible oil solutions obeys approximately lst order kinetics.

\section{Effect of Injection Volume}

Injection volume is one of the important factors which influence drug absorption. Fig. 3 shows the effect of the injection volume on the absorption time profiles following intramuscular administration of PAAB in SO. The slope of these profiles increased gradually with decreasing injection volume. The quantitative relationship is discussed later.

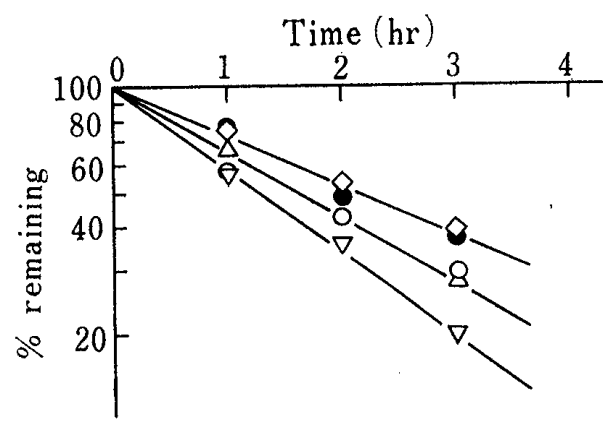

Fig. 3. Effect of Injection Volume on Intramuscular Absorption of PAAB from SO Solution

Key: $-\nabla-, 2.5 \mu 1 ;-\bigcirc-, 5 \mu 1 ;-\triangle-, 10 \mu 1 ;-\diamond-, 50$ $\mu 1 ;-0-, 100 \mu \mathrm{l}$. Each point represents the mean of 4 or 5 experiments. $C_{0}: 5 \mathrm{mg} / \mathrm{ml}$.

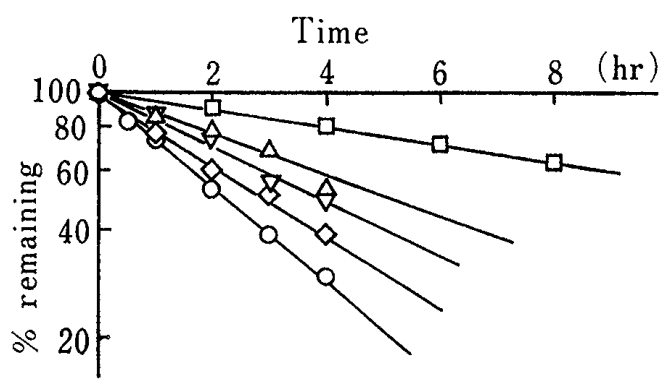

Fig. 4. Comparison of Intramuscular Absorption of PAAB from Various Oily Solutions

Key: $-\square-$, DES; $-\triangle-$, Mig; $-\nabla-$, CO; $-\diamond-$, IPM; $-\mathrm{O}^{-}, \mathrm{SO}$. Each point represents the mean of 4 or 5 experiments. $C_{0}, 5 \mathrm{mg} / \mathrm{ml} ; V_{0}, 0.05 \mathrm{ml}$.

\section{Comparison of Absorption Rates from Various Oily Solvents}

In parenteral preparations for animal experiments, oily solvents other than sesame oil are used with or without some adjuvants. Fig. 4 compares the intramuscular absorption rates of PAAB in five oily solvents, DES, Mig, CO, IPM and SO. Each semilogarithmic plot was nearly linear with a different slope. Similar results were also obtained for other compounds, PHAB and OAAT. The major factors which resulted in the remarkable differences in the absorption rate from different vehicles will be discussed below.

\section{Discussion}

\section{Kinetics of Drug Absorption}

Visual observation throughout all our absorption experiments confirmed that dye solution injected intramuscularly was confined to the planes of fascia or connective tissues surrounding muscles, did not spread as extensively along the fascial septums as did the aqueous systems, and formed a depot which took a flat shape similar to a pod. The geometrical change of the oily depot was more abrupt within a short period during and immediately after injection but gradually became less with time. Our observations were similar to those of Scaffer. ${ }^{11)}$

When a practically water-insoluble drug in a water-immiscible oil solution is injected intramuscularly, two possible routes for the absorption of the drug component may be considered: (i) absorption by direct transport of small oil droplets containing the drug and (ii) 
absorption by the same mechanism as an aqueous solution after drug release from the oily phase into the aqueous phase. The absorption data for the oily solvent (SO) shown in Table II imply that for several hours after injection, SO was scarcely absorbed. Thus, the second route (ii) is expected to be the major one for drugs which are more quickly absorbed than the oily solvent.

Thus, assuming the driving force for drug transport to be diffusion, a model was proposed to clarify the absorption process kinetically. Fig. 5 shows the absorption process of the drug component diagrammatically with a two-dimensional model. The broken lines represent the concentration gradient along the distance axis perpendicular to the interface (i) between the oily and aqueous phases. First, the drug molecule is transported in the oily phase (I) to the interface (i), where it is then distributed into another phase (II). Subsequently, the drug molecule in the aqueous phase (II) diffuses with or without the aid of body fluid flow through the biological matrix, including cell membranes or intercellular spaces of connective, muscle and vascular tissues, into the blood or lymph stream. The shape of the oily depot in the injection site may fluctuate continually in response to the motion of the surrounding muscles and this may cause some convection flow, that is, agitation within the oily depot phase (I). In the aqueous phase (II), the flow of body fluid is expected to be vigorous, especially in the region adjacent to vascular systems (III). If we assume on the basis of these considerations that the rate-limiting process of drug absorption may exist in the diffusion in both phases close to the interface (i), the fluxes of the drug molecules per unit time, $J_{0}$ and $J_{\mathrm{a}}$, are given by the following equations according to Fick's diffusion law:

$$
\begin{array}{ll}
J_{\mathrm{o}}=-D_{\mathrm{o}}\left(\frac{\partial C}{\partial X}\right)_{X=X_{1}} \quad\left(0<X<X_{\mathrm{i}}\right) \\
J_{\mathrm{a}}=-D_{\mathrm{a}}\left(\frac{\partial C}{\partial X}\right)_{X=X_{1}} \quad\left(X>X_{\mathrm{i}}\right)
\end{array}
$$

where $D_{\mathrm{o}}$ and $D_{\mathrm{a}}$ represent diffusion coefficients in the oily and aqueous phases, respectively, and $(\partial C / \partial X)$ signifies the concentration gradient of the drug in the oily $\left(\mathrm{O}<X<X_{1}\right)$ or aqueous $\left(X>X_{\mathrm{i}}\right)$ phase. Assuming that the thickness of the diffusion layer may be approximately regarded as constant, Eqs. 1 and 2 become:

$$
\begin{aligned}
& J_{\mathrm{o}}=k_{\mathrm{o}}\left(C_{\text {ol1 }}-C^{\prime}{ }_{\text {oil }}\right) \\
& J_{\mathrm{a}}=k_{\mathrm{a}}\left(C_{\mathrm{a}}^{\prime}-C_{\mathrm{b}}\right)
\end{aligned}
$$

where $k_{\mathrm{o}}$ and $k_{\mathrm{a}}$ are defined as parameters related to transport in the oily and aqueous phases, respectively, and $C_{\mathrm{o} i 1}$ and $C_{\mathrm{b}}$ repesent concentrations in the oil bulk phase and blood, respec-

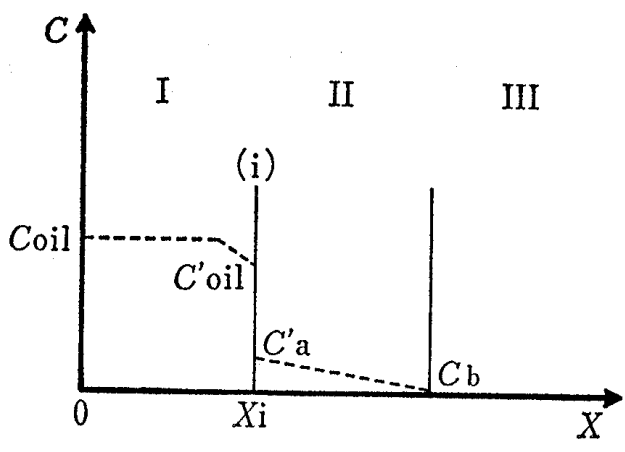

Fig. 5. Model for Intramuscular Drug Absorption from Water-immiscible Oil Vehicle

I : Oily depot phase,

II : Aqueous body fluid phase containing various tissues adjacent to oily depot,

III: Blood or lymph.

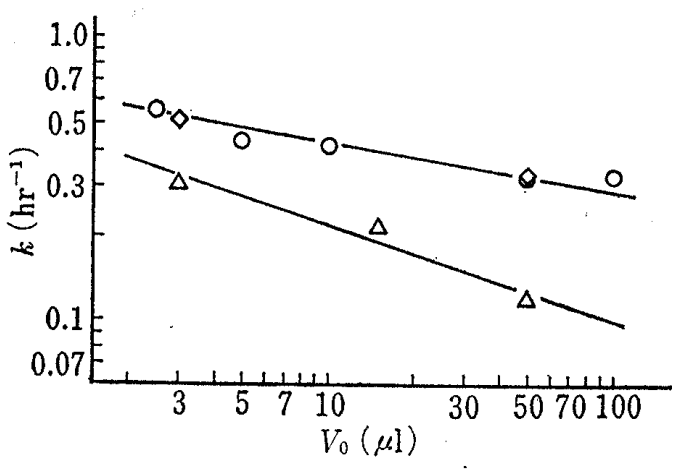

Fig. 6. Relation between Absorption Rate Constant $(k)$ and Injection Volume $\left(V_{0}\right)$

Key: $-\mathrm{O}$, intact, $m$. gastrocnemius $\left(C_{0}, 5 \mathrm{mg} / \mathrm{ml}\right)$; $-\diamond$-, intact, $m$. rectus femoris $\left(C_{0}, 10 \mathrm{mg} / \mathrm{ml}\right) ;-\triangle$, , anesthetized, $m$. gastrocnemius $\left(C_{0}, 10 \mathrm{mg} / \mathrm{ml}\right)$.

Test compound, PAAB; vehicle, SO. 
tively; $C^{\prime}{ }_{\text {oil }}$ and $C^{\prime}{ }_{a}$ mean the concentrations in the oily and aqueous phases at $X=X_{\mathrm{i}}$, respectively. Under the condition that $C_{\mathrm{a}}^{\prime} \gg C_{\mathrm{b}}$, Eq. 4 can be approximated as:

$$
J_{\mathrm{a}}=k_{\mathrm{a}} C_{\mathrm{a}}^{\prime}
$$

Assuming that the distribution equilibrium of the drug molecule at the interface is reached quickly compared with its transport, the distribution coefficient $\left(K_{\mathrm{i}}\right)$ at the interface is defined by

$$
K_{\mathrm{i}}=C^{\prime}{ }^{\prime}{ }^{1} / C^{\prime}{ }_{\mathrm{a}}
$$

After a quasi-steady-state is reached at time $\tau$, the following relationship is established:

$$
-\mathrm{d} W / \mathrm{d} t=A J_{\mathrm{o}}=A J_{\mathrm{a}} \quad(t>\tau)
$$

where $W$ represents the amount of drug in the oily depot phase and $A$ is the surface area of the oily depot. $W$ is given by the volume of the oily depot, $V$, and the drug concentration in it, $C_{\text {oil }}$, according to the following equation:

$$
W=C_{\text {oil }} V
$$

From Eqs. 3, 5, 6, 7 and 8, the following equation can be derived:

$$
\frac{\mathrm{d} W}{\mathrm{~d} t}=-\left[\frac{k_{\circ} A}{V\left(1+\frac{k_{\mathrm{o}}}{k_{\mathrm{a}}} K_{\mathrm{i}}\right)}\right] W
$$

Since the absorption of oil itself is very slow compared with that of the drug component, as shown in Tables I and II, $V$ can be regarded as equal to the injection volume $V_{\mathbf{0}}$ for several hours after administration. Under the condition that the change in the surface area of the oily depot, $A$, is sufficiently small, Eq. 9 shows the 1st order kinetics and can be integrated. When lag time $\tau$ is sufficiently small compared with the absorption time scale, the following relations are readily derived:

$$
\begin{aligned}
& \ln \left(W / W_{0}\right)=-k t \\
& k=\frac{k_{0} A}{V_{0}\left(1+\frac{k_{0}}{k_{\mathrm{a}}} K_{\mathrm{i}}\right)}
\end{aligned}
$$

where $W_{0}$ represents $W$ at $t=0$, that is, the dose, and $k$ is defined as a lst order absorption rate constant. For the model drugs used in this study, it was observed that a large portion of the amount remaining in the injection site was localized within the oily depot phase. Accordingly, the value of $W / W_{0}$ for these drugs is nearly equal to the residual fraction determined by the absorption experiment described above.

The experimental results shown in Fig. 2 indicate that the absorption obeys lst order kinetics. Therefore, provided that the assumptions and conditions in the above consideration based on the model are valid, the apparent lst order absorption rate constant experimentally obtained is governed by $A, V_{0}, k_{\mathrm{o}}, k_{\mathrm{a}}$ and $K_{\mathrm{i}}$.

\section{Relationship between Absorption Rate Constant and Injection Volume}

Some papers have reported that the intramuscular absorption rate of a drug in aqueous ${ }^{10,12}$ or oily solution ${ }^{5}$ might decrease with increasing injection volume. However, the quantitative relationship between absorption rate and injection volume or the influence of anesthesia and the injection site on it has not been completely elucidated. Fig. 6 shows a plot of the lst order absorption rate constant $(k)$ against injection volume $\left(V_{0}\right)$ on a log-log scale. In this figure, the data obtained from Fig. 3 are indicated by open circles. This plot gave a straight line with a slope of -0.14 (correlation coefficient, $r=-0.95$ ). The data from intramuscular injection into the other site, the m. rectus femoris, in intact rats (indicated as open squares in Fig. 6) gave an almost identical straight line. In anesthetized rats, as shown by open. 
triangles in Fig. 6, the absorption rate was remarkably slower compared with the corresponding data in the unanesthetized (intact) rats. A plot of $\log k$ versus $\log V_{0}$ gave a similar straight line but with a larger slope, $-0.32(r=-0.97)$.

Eq. 11 means that the absorption rate constant $k$ is proportional to the term $A / V_{0}$, which depends on the injection volume $\left(V_{0}\right)$. The term $A / V_{0}$ is proportional to $V_{0}{ }^{-1 / 3}$ when the oily depot retains a similar shape or dimensions for the change in $V_{0}$, and it is constant when the oily depot expands two-dimensionally at a constant thickness with increasing $V_{0}$. The relation $k \propto V_{\mathbf{0}}{ }^{-\mathbf{0 . 3 2}}$ observed in anesthetized rats may correspond to the former case, while $k \propto V_{0}-0.14$ observed in unanesthetized rats may correspond to an intermediate case between the two cases mentioned above. It seems possible that in intact rats, unlike anesthetized rats, the oily depot cannot retain a similar shape or dimensions for an increase in $V_{0}$ because of the motions of the more tensioned muscle fibers around it.

\section{Effect of Vehicle Viscosity and Distribution Coefficient}

Fig. 4 compares the intramuscular absorption rates of PAAB from various oily solvents. The difference in absorption rate in the different vehicles can be attributed to the diffusional resistance in the oily phase and/or the distribution coefficient between the oil and the body fluid. The absorption rate constant given in Eq. 11 involves the parameters $k_{o}$ and $K_{1}$. It is apparent that $k_{\mathrm{o}}$ is inversely proportional to the viscosity of the oil vehicle and $K_{\mathrm{i}}$ has a positive relation with the distribution coefficient $(K)$ determined in vitro. To evaluate the degree of contribution of viscosity $(\eta)$ and the distribution coefficient $(K)$ to absorption, these factors are summarized in Table III and compared with the absorption rate constant $(k)$ obtained experimentally. From this table, it is evident that for all compounds tested, $k$ increases with a decrease in $K$ but depends little on $\eta$.

TABLE III. List of Values of Viscosity $(\eta)$ of Oil Vehicles, Distribution Coefficient $(K)^{a}$ and Absorption Rate Constant $(k)^{b)}$

\begin{tabular}{|c|c|c|c|c|c|c|c|}
\hline \multirow{2}{*}{ Oil } & \multirow{2}{*}{$\eta(\mathrm{c} . \mathrm{p})}$. & \multicolumn{2}{|r|}{ PAAB } & \multicolumn{2}{|r|}{ PHAB } & \multicolumn{2}{|r|}{ OAAT } \\
\hline & & $K^{\prime}$ & $k\left(\mathrm{hr}^{-1}\right)$ & $K$ & $k\left(\mathrm{hr}^{-1}\right)$ & $K$ & $k\left(\mathrm{hr}^{-1}\right)$ \\
\hline $\mathrm{SO}$ & 35 & 1200 & $0.329(0.029)$ & 1300 & $0.431(0.038)$ & 13400 & $0.0775(0.0066)$ \\
\hline IPM & 3.6 & 1700 & $0.241(0.022)$ & & & & \\
\hline $\mathrm{CO}$ & 286 & 2500 & $0.193(0.011)$ & & & & \\
\hline Mig & 15 & 2900 & $0.146(0.009)$ & 3500 & $0.140(0.013)$ & 27000 & $0.0340(0.0025)$ \\
\hline DES & 3.9 & 9900 & $0.058(0.007)$ & 15000 & $0.0460(0.0053)$ & 75000 & $0.0179(0.0029)$ \\
\hline
\end{tabular}

a) Distribution coefficient between oil vehicle and $0.9 \%(\mathrm{w} / \mathrm{v}) \mathrm{NaCl}$ aqueous solution at $37^{\circ}$.

b) lst order rate constant. All $k$ values were estimated by the least-squares method using the data during the first $8 \mathrm{hr}$ after injection, and are tabulated together with the standard errors (S.E.) in parentheses.

If $K_{\mathrm{i}}$ is sufficiently large and therefore the relation $\left(k_{\mathrm{o}} / k_{\mathrm{a}}\right) K_{\mathrm{i}} \gg 1$ holds, Eq. 11 can be approximated as

$$
k=\frac{A k_{\mathrm{a}}}{V_{0} K_{\mathrm{i}}}
$$

This equation means that $k$ is independent of $k_{\mathrm{o}}$, that is, independent of the viscosity of the oil vehicle, and is inversely proportional to $K_{\mathrm{f}}$ if the other parameters are fixed. If this equation is the case, a plot of $k$ against $K_{\mathrm{i}}$ on a log-log scale should give a straight line with a slope of -1 . Fig. 7 shows this plot for the three compounds presented in Table III with the $K$ values determined in vitro. Each plot gave a good linear relationship. The regression line of each compound, as given in the legend, has a slope close to -1 but of a slightly smaller absolute value. These results indicate that from a macroscopic point of view, Eq. 12 is approximately valid and can reasonably explain the intramuscular absorption phenomena of practical- 


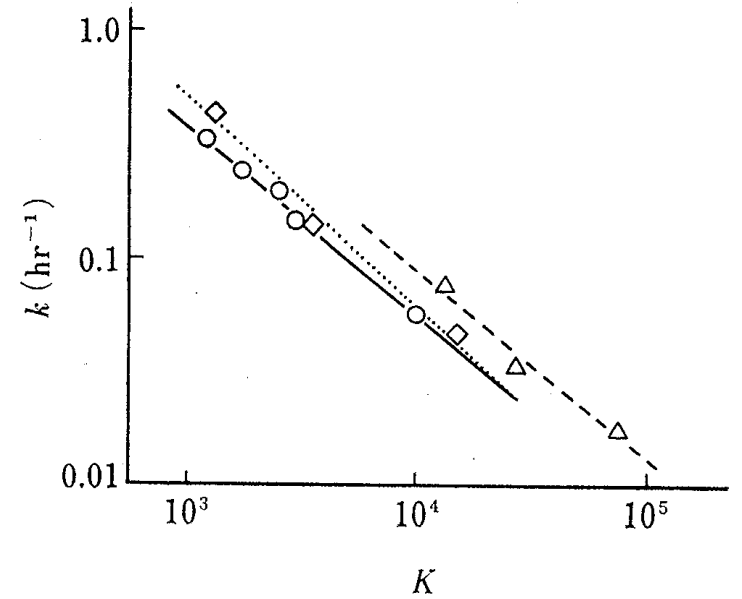

Fig. 7. Relation between Absorption Rate Constant $(k)$ and Distribution Coefficient $(K)$

Key: $-\bigcirc-$, PAAB; $-\diamond---$, PHAB; $-\triangle--$, OAAT.

Regression line $(y=\log k, x=\log K):$ PAAB, $y=-0.82$

$x+2.05 \quad(r=-0.996) ;$ PHAB, $y=-0.90 x+2.41 \quad(r=$

$-0.994)$; OAAT, $y=-0.84 x+1.76(r=-0.984)$.

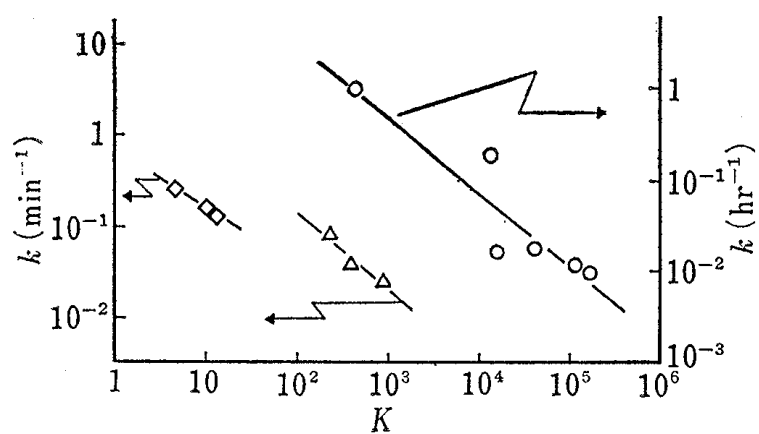

Fig. 8. Plots of $k$ versus $K$ from Literature Data

$-0-$ : Testosterone and its esters in ethyl oleate vehicle ${ }^{13)}$ $y=-0.81 x+2.12(r=-0.92)$

$-\triangle-:$ Testosterone in methyl oleate, tributyrin and diethyl phthalate vehicles ${ }^{5)} \quad y=-0.86 x+0.88(y=-0.96)$

$-\diamond$-: Methylisonicotinate in the three vehicles noted above $^{5)} y=-0.65 x-0.14(r=-0.99)$

ly water-insoluble drugs in water-immiscible oil solutions.

Fig. 8 gives the same plot as Fig. 7 for data from the literature. ${ }^{5,13)}$ As with our results, each of these plots also gave a single straight line with a slope close to -1 but of a slightly smaller absolute value. These findings suggest that the linear relationship shown in Fig. 7 holds not only for systems having a $K$ value smaller than those in our study but also for different compounds in homologous series in the same vehicle. The latter case implies that the difference in $k_{\mathrm{a}}$ in Eq. 12 among different compounds of similar molecular weight in a homololgous series may not be very large. The proximate position of the three regression lines in Fig. 7 supports this implication.

\section{Estimation of Absorption Rates of Other Drugs from Oily Solutions}

Fig. 7 showed that the regression lines of three compounds different in lipophilicity were proximal to each other, and the combined data gave the following regression equation: $\log k=$ $-0.73 \log K+1.76(r=-0.98)$. It was expected that this relationship could be applied for predicting the absorption rate constant $(k)$ of other drugs from the $K$ value. To examine this possibility, testosterone (TS), epitiostanol and 1-phenylazo-2-naphthylamine (PANA), having different $K$ values but molecular weights similar to those of the three compounds presented in Fig. 7, were selected as test materials. The results are summarized in Table IV. For PANA, the observed $k$ value was considerably larger than the predicted value. This difference may be accounted for by the exceedingly larger $K$ value, since the release of such a compound

TABLE IV. Comparison of Predicted and Experimental Intramuscular Absorption Rate Constants $(k)^{a)}$

\begin{tabular}{|c|c|c|c|c|}
\hline \multirow{2}{*}{ Compound } & \multirow{2}{*}{$\mathrm{M} . \mathrm{W} \cdot{ }^{b\rangle}$} & \multirow{2}{*}{$K^{c)}$} & \multicolumn{2}{|c|}{$k\left(\mathrm{hr}^{-1}\right)$} \\
\hline & & & Predicted ${ }^{d)}$ & Experimental (S.E.) \\
\hline TS & 288 & 130 & 1.67 & $1.38 \quad(0.13)$ \\
\hline Epitiostanol & 311 & 12500 & 0.060 & $0.0693(0.0071)$ \\
\hline PANA & 247 & 382000 & 0.0050 & $0.0134(0.0013)$ \\
\hline
\end{tabular}

a) SO solution; $\mathrm{C}_{0}, 5 \mathrm{mg} / \mathrm{ml} ; V_{0}, 0.05 \mathrm{ml}$.

b) Molecular weight.

c) Distribution coefficient between $\mathrm{SO}$ and $0.9 \% \mathrm{NaCl}$ aqueous solution.

d) Predicted by using the regression equation obtained from all the data points in Fig. $7: \log k=-0.73 \log K+1.76$. 
from the oily phase is exceedingly slow and therefore the contribution of drug absorption route $(i)$ is not negligible as inferred from the data in Table II. For TS and epitiostanol, the observed and predicted $k$ values were in reasonable agreement. These results suggest that further similar predictions will be possible for other compounds.

\section{Absorption Rate from Oily Suspensions}

Increasing the concentration of a drug in an oily solution produces an oily suspension. Oily suspensions are commonly used as prolonged-release injections. However, very little work has been done on the drug absorption rate process after intramuscular administration of oily suspensions. It thus seems useful to relate the drug absorption rate in oily suspension with that in oily solution.

In oily suspensions, $C_{\text {oil }}$ in Eq. 3 equals the saturated concentration $C_{\text {s, oil }}$. Taking into account this relationship, an equation representing the rate of loss of the drug amount from the injection site, $\mathrm{d}(W)_{\text {susp }} / \mathrm{d} t$, can be readily derived from Eqs. 3, 5, 6 and 7:

$$
\frac{\mathrm{d}(W)_{\text {susp }}}{\mathrm{d} t}=-\frac{f A k_{\mathrm{o}} C_{\mathrm{s}, \mathrm{oi} I}}{1+\frac{k_{\mathrm{o}}}{k_{\mathrm{a}}} K_{\mathrm{i}}}
$$

where a factor $f$ is introduced to correct for the difference in geometry of the depot between oily solution and suspension at the same injection volume. Substitution of the relationship $k_{\mathrm{o}} A /\left(1+k_{\mathrm{o}} K_{\mathrm{i}} / k_{\mathrm{a}}\right)=k V_{0}$, derived from Eq. 11, into the right-hand side of Eq. 13 and subsequent integration from time zero to $t$ yield the following equation:

$$
(W)_{\text {susp }}=\left(W_{0}\right)_{\text {susp }}-k f C_{\mathrm{s}, \text { oil }} V_{0} t \quad\left(0<t<\frac{\left(W_{0}\right)_{\text {susp }}-C_{\mathrm{s}, \text { oil }} V_{0}}{k f C_{\mathrm{s}, \text { oil }} V_{0}}\right) \quad \text { (Eq. 14) }
$$

where $\left(W_{0}\right)_{\text {susp }}$ is the drug amount injected (i.e., dose). Dividing both sides of Eq. 14 by $\left(W_{0}\right)_{\text {susp }}$, employing the relationship $\left(W_{0}\right)_{\text {susp }}=C_{0 \text {, susp }} V_{0}$ and setting $n$ equal to $C_{0 \text {, susp }} / C_{\mathrm{s} \text {,oil }}$, we can write Eq. 14 as follows:

$$
\begin{aligned}
& \left(W / W_{0}\right)_{\text {susp }}=1-\phi t \\
& \phi=f k / n
\end{aligned}
$$

where $\phi$ represents a zero-order absorption rate constant for the drug in oily suspension and $k$ is a 1st order absorption rate constant for the solution of the same drug and the same oily solvent at the same injection volume and at the same injection site. These equations mean that under conditions where a sufficient amount of suspended particles remains in the oily depot phase, the absorption of the drug proceeds approximately according to zero-order kinetics, and its rate constant is inversely proportional to $n$ (accordingly, to $C_{0}$,susp or dose) if the correction factor $f$ is constant.

Fig. 9 shows the absorption time profiles of testosterone (TS) from oily suspensions of different vehicles, SO, IPM and Mig under the same conditions. The plot of $\left(W / W_{0}\right)_{\text {susp }}$ versus time on rectilinear coordinates for the three suspensions demonstrates that Eq. 15 is experimentally acceptable for an early period after intramuscular injection, though small upward deviations occur with the passage of time. The results appearing in this figure, that the slope of the initial linear segment of these curves depended little on the oil vehicle, can be explained as an apparent phenomenon caused by the following conditions: the concentration of TS dissolved in the oily depot phase at the interface, $C^{\prime}{ }_{\text {oll }}$, approaches $C_{\mathrm{s}, \text { oil }}$ in each oil vehicle and hence the concentration of TS in the aqueous phase, $C^{\prime}{ }_{a}$, is close to $C_{\mathrm{s}, \mathrm{a}}$ (solubility in body fluids surrounding the oily depot) for all three suspensions. ${ }^{14)}$

If the range of the correction factor $f$ in Eq. 16 is known, the zero-order absorption rate constant during an early period after injection can be roughly estimated from the $k$ value. To evaluate $f$, various absorption experiments were done using TS suspensions of different $C_{0 \text {, susp }}$ and particle sizes. The apparent zero-order rate constants $\phi_{\mathrm{app}}$ (obtained from the linear 


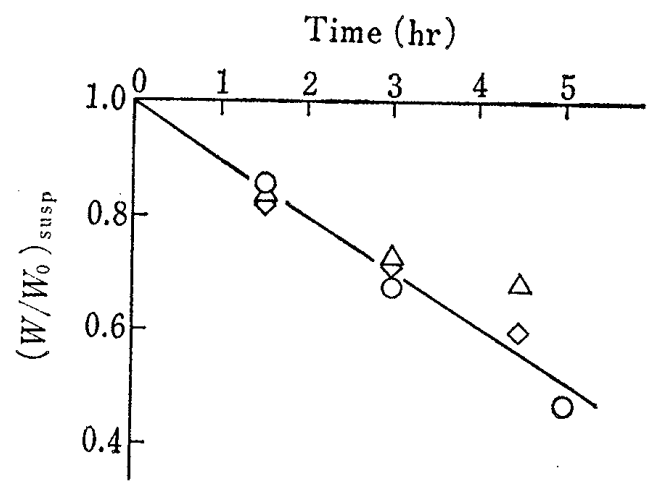

Fig. 9. Absorption Time Profiles of TS from Various Oily Suspensions in Different Media after Intramuscular Injection

Key: $\bigcirc$, SO $(n=6.0) ; \triangle$ IPM $(n=5.3) ; \diamond, \mathrm{Mig}$ $(n=3.1)$. Each point represents the mean of at least 4 experiments. $C_{\theta \text {, susp }} 50 \mathrm{mg} / \mathrm{ml} ; V_{0}, 0.05 \mathrm{ml}$; particle size, $37-74 \mu \mathrm{m}$.

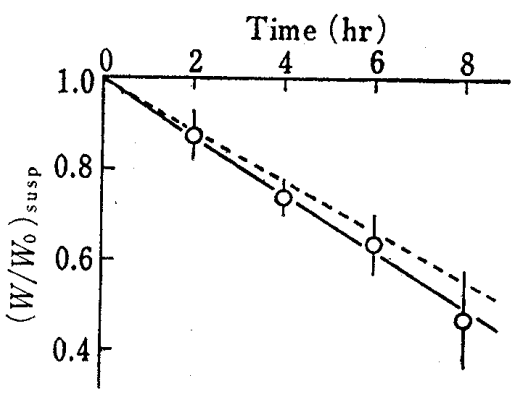

Fig. 10. Predicted and Observed Absorption Curves of PAAB Oily Suspension after Intramuscular Injection

Key: $-\mathrm{O}^{-}$, observed (each point represents the mean of 4 experiments); broken line, predicted using values of $0.33 \mathrm{hr}^{-1}, 2.9$ and 0.5 for $k, n$ and $f$ respectively. $C_{0 . \text { susp }}, 165 \mathrm{mg} / \mathrm{ml} ; V_{0}, 0.05 \mathrm{ml}$; vehicle, SO; particle size, $74-149 \mu \mathrm{m}$.

TABLE V. Apparent Zero-order Absorption Rate Constants $\left(\phi_{\text {app }}\right)$ and Resultant $f$ Values for Testosterone Suspensions of Different Initial Concentrations $\left(C_{0, \text { susp }}\right)$ and Particle Sizes $\left.{ }^{a}\right)$

\begin{tabular}{ccccc}
\hline \hline $\begin{array}{c}\text { Particle size } \\
(\mu \mathrm{m})\end{array}$ & $\begin{array}{c}C_{\mathbf{0}, \mathrm{susp}^{b)}} \\
(\mathrm{mg} / \mathrm{ml})\end{array}$ & $n^{c)}$ & $\begin{array}{c}\phi_{\mathrm{app}}(\mathrm{S} . \mathrm{E} .) \\
\left(\mathrm{hr}^{-1}\right)\end{array}$ & $f^{d)}$ \\
\hline $74-149$ & 24.9 & 3 & $0.240(0.022)$ & 0.52 \\
& 49.8 & 6 & $0.105(0.009)$ & 0.46 \\
$37-74$ & 83.0 & 10 & $0.056(0.005)$ & 0.41 \\
& 24.9 & 3 & $0.260(0.020)$ & 0.57 \\
& 49.8 & 6 & $0.100(0.007)$ & 0.43 \\
& 83.0 & 10 & $0.060(0.004)$ & 0.43 \\
\hline
\end{tabular}

a) Medium, SO; injection volume, $0.05 \mathrm{ml}$.

b) Initial TS concentration.

c) Calculated as $n=C_{0, \text { susp }} / C_{\mathrm{s} \text {, oi1 }}$ using a value of $8.3 \mathrm{mg} / \mathrm{ml}$ for $C_{\mathrm{s}, \text { oil }}$

d) Calculated as $f=n \phi_{\mathrm{app}} / k$ using a value of $1.38 \mathrm{hr}^{-1}$ for $k$ (from Table IV).

segrinerits of absorption curves for the first $2 \mathrm{hr}$ ) and the correction factor $f$, calculated by means of Eq. 16 from $\phi_{\mathrm{app}}, n$ and $k$, are summarized in Table $\mathrm{V}$, together with the experimental conditions. For initial concentrations of the drug in the solid state ranging from 16.6 to 74.7 $\mathrm{mg} / \mathrm{ml}$ in the particle size range of $37-149 \mu \mathrm{m}$, the value of $f$ varied little $(0.41-0.57$; mean, about 0.5 ). This implies that $\phi$ for other oily suspensions with characteristics similar to those presented here would be predictable in rough approximation from $k$ and $n$ by using the above $f$ value. Fig. 10 compares the predicted and observed absorption curves for the PAAB suspension in SO (particle size, $74-149 \mu \mathrm{m} ; C_{0 \text {, susp }}, 165 \mathrm{mg} / \mathrm{ml}$ ). The values for $k, n$ and $f$ used in this prediction were $0.33 \mathrm{hr}^{-1}$ (from Table III), 2.9 and 0.5 , respectively. The good agreement of the two curves indicates that this prediction method should also be useable for other suspension systems.

For systems in which the drug component is absorbed more quickly than the oil vehicle and in which the drug or oil vehicle is physiologically inactive to the surrounding tissues, the drug absorption behavior can be reasonably explained in physicochemical terms. However, if the drug can hardly be released from the oil vehicle or if the vehicle exerts some local effect, additional factors such as absorption or metabolism of the vehicle itself and physiological changes at the injection site should be taken into consideration in attempting to understand 
the drug absorption phenomena. Further investigations will be necessary to evaluate the contribution of these factors to drug absorption.

Acknowledgement The authors are indebted to Mrs. F. Tanaka and Mrs. J. Kagawa for skillful technical assistance and also to Prof. J. Watanabe, Nagoya City University, for helpful discussions. The authors also wish to thank Prof. M. Nakagaki, Kyoto University, for his valuable comments on the manuscript.

\section{References and Notes}

1) a) J. Schou, Pharmacol. Rev., 13, 441 (1961); b) J.G. Wagner, J. Pharm. Sci., 50, 383 (1961); c) B.E. Ballard, ibid., 57, 357 (1968).

2) a) K. Kakemi, H. Sezaki, K. Okumura, and S. Ashida, Chem. Pharm. Bull., 17, $1332(1969)$; b) K. Kakemi, H. Sezaki, K. Okumura, C. Takada, and S. Furusawa, ibid., 19, 2058 (1971); c) K. Kakemi, H. Sezaki, K. Okumura, H. Kobayashi, and S. Furusawa, ibid., 20, $443(1972)$; d) K. Okumura, H. Sezaki, and K. Kakemi, ibid., 20, 1607 (1972); e) H. Kobayashi, T. Nishimura, K. Okumura, S. Muranishi, and H. Sezaki, J. Pharm. Sci., 63, 580 (1974).

3) a) E. Secher-Hansen, H. Langgard, and J. Schou, Acta Pharmacol. Toxicol., 25, 162, 290 (1967); b) Idem, ibid., 26, 9 (1968); c) B.E. Ballard and E. Menczel, J. Pharm. Sci., 56, $1476(1967)$; d) R.H. Levy and M. Rowland, J. Pharmacokin. Biopharm., 2, 313, 337 (1974).

4) a) J. Dekanski and R.N. Chapman, Brit. J. Pharmacol., 8, 271 (1953); b) J.C. Bauernfeind and H.L. Newmark, Bull. Parenteral Drug Assoc., 24, $169(1970) ; \quad c)$ B.H. Fusa and K.E. Avis, ibid., 26, 1 (1972).

5) T. Tanaka, H. Kobayashi, K. Okumura, S. Muranishi, and H. Sezaki, Chem. Pharm. Bull., 22, 1275 (1974).

6) Private communications from Drs. R. Konaka and T. Mizuta of Shionogi Research Laboratories (Sagisu 5-12-4, Fukushima-ku, Osaka).

7) $\mathrm{Al}_{2} \mathrm{O}_{3}$, the same as described above; silica gel, Wakogel Q-23 (Wako Pure Chemicals Industries, Osaka).

8) J.N. Bollinger, J. Pharm. Sci., 59, 1084, 1088 (1970).

9) M. Hashida, M. Egawa, S. Muranishi, and H. Sezaki, J. Pharmacokin. Biopharm., 5, 225 (1977).

10) R.B. Sund and J. Schou, Acta Pharmacol. Toxicol., 21, 313 (1964).

11) L.W. Scaffer, Arch. Dermatol., 19, 347 (1929).

12) G.F. Warner, E.L. Dobson, N. Pace, M.E. Johnston, and C.R. Finney, Circulation, 8, 732 (1953).

13) K.C. James, P.J. Nicholas, and M. Roberts, J. Pharmac. Pharmacol., 21, 24 (1969).

14) In this case, $K_{\mathrm{i}}$ is nearly equal to $C_{\mathrm{s}, \mathrm{oi} 1} / C_{\mathrm{s}, 2}$. Substitution of $k$ in Eq. 12 into Eq. 16 and rearrangement result in the following relationship. $\phi \fallingdotseq \frac{f A k_{\mathrm{a}}}{V_{0} K_{\mathrm{i}}} / \frac{C_{0, \mathrm{susp}}}{C_{\mathrm{s}, \text { oil }}}=\frac{f A k_{\mathrm{a}} C_{\mathrm{s}, \mathrm{a}}}{V_{0} C_{0} \text {, susp }}\left(=\right.$ nearly constant at fixed $V_{0}$ and $C_{0}$, susp if the factor $f$ changes little). 